\title{
Protocolo Avaliativo de Iniciação Esportiva em Cadeira de Rodas: uma alternativa de avaliação para pessoas com deficiência física
}

Flávio Anderson Pedrosa de Melo ${ }^{a, b *}$, Mey de Abreu van Munster ${ }^{a}$

\section{Palavras Chave:}

Educação Física; Iniciação Esportiva; Deficiência Física; Avaliação.

\section{Keywords:}

Physical Education; Sports Initiation; Physical Disability; Evaluation.

\begin{abstract}
RESUMO
O estudo teve como objetivo apresentar o Protocolo Avaliativo de Iniciação Esportiva em Cadeira de Rodas - PAIE-CR e verificar em quais áreas dos componentes motores (habilidades motoras fundamentais e capacidades físicas), um programa de iniciação esportiva em cadeira de rodas exerceu influência, antes e após a intervenção proposta. A intervenção foi composta por 24 sessões. Participaram desse estudo três crianças ( 6 a 10 anos) com deficiência física, comprometimento nos membros inferiores. O PAIE-CR é composto por nove testes divididos em quatro grupos de componentes: estabilização; locomoção; manipulação e combinados. Acredita-se que o instrumento oportuniza o acompanhamento e permite avaliar os componentes inerentes à iniciação esportiva em cadeira de rodas do educando.
\end{abstract}

\begin{abstract}
The purpose of this study was to present a Wheelchair Sportive Initiation Protocol (WSIP). In addition we verified the effects of a sport initiation program in the target motor components fields (considering the main motor skills and capabilities for sport initiation in wheelchair). The intervention was carried out over 24 sessions. Three children aged 6-10 years with physical disability and lower limbs impairment participated in this study. The WSIP is composed for nine tests divided in four components groups: stabilization; locomotion; manipulation and matched. We believe that the instrument enables the assessment as well as follow-up of the learner with physical disability. Moreover, the WSIP allows assessing the components regarding the sport initiation in wheelchair.
\end{abstract}

\section{RESUMEN}

El estudio en cuestión tuvo como objetivo presentar el Protocolo de Evaluación de Iniciación Deportiva en Silla de Ruedas - PEIDSR y verificar en qué áreas de las habilidades motoras básicas y fundamentos deportivos, el programa de iniciación deportiva en silla de ruedas ejerció mayor o menor influencia, antes y después de la intervención propuesta. Se trata de una investigación de campo del tipo cuantitativo-descriptivo, volcada al estudio de evaluación de programa. En este estudio participaron tres niños (6 a 10 años) con discapacidad física, comprometimiento en los miembros inferiores. Los parámetros de evaluación utilizados, en la aplicación del PEIDSR demostró que el mismo logró atender las necesidades del estudio, ya que fue posible evaluar cada una de las variables del programa y del estudio.

\footnotetext{
a Universidade Federal de São Carlos, São Carlos, SP, Brasil.

${ }^{\mathrm{b}}$ Instituto Federal de Educação, Ciência e Tecnologia de Alagoas, Maceió, AL, Brasil.
} 


\section{INTRODUÇÃO}

Nos dias atuais, a ampliação de iniciativas políticas, educacionais e científicas voltadas à pessoa com deficiência tem possibilitado o envolvimento dessas em diferentes contextos, inclusive o esportivo. As atividades físicas e esportivas desenvolvidas por pessoas com deficiências, necessitam ser estimuladas, se possível, desde a infância, buscando proporcionar vivências motoras que serão fundamentais para a iniciação ao esporte, com o intuito de identificar ferramentas para avalia-las de maneira adequada.

Um programa de iniciação esportiva em cadeira de rodas para crianças com deficiência física deve considerar as características individuais de cada um dos participantes, considerando os interesses, as expectativas e o potencial da criança, independentemente dos níveis de apoio que o mesmo necessita (Gorgatti e Costa, 2013; Mauerberg-deCastro, 2005; Melo e Munster, 2016). A partir da identificação de tais necessidades específicas do integrante, o professor estará mais capacitado a planejar o programa de ensino de maneira a aperfeiçoar as potencialidades do aluno e suas habilidades motoras e capacidades físicas e de compreensão dos aspectos a serem controlados e avaliados (Melo e Munster, 2016).

As habilidades na cadeira de rodas são passíveis de melhoria por meio de treinamento, principalmente quando possuem um formato estruturado (Giesbrecht et al, 2017). Existem programas de treinamento de habilidades em cadeira de rodas que possibilitam efeitos benéficos a diferentes populações e contextos (Dalhousie University, 2018; Ozturk e Ucsular, 2011; MacPhee et al, 2004). Nesse sentido, torna-se essencial obter ferramentas para acompanhamento e avaliação dos participantes.

$A$ avaliação tem variadas funções em meio à área da Educação Física Adaptada, o processo avaliativo tornase imprescindível para a verificação de necessidades especiais, bem como no estabelecimento de uma base de instrução (Winnick e Short, 2001). Além disso, pode auxiliar no monitoramento de alterações desenvolvimentistas, na identificação de atrasos e na obtenção de esclarecimentos sobre as estratégias a serem utilizadas (Gorla e Campos, 2013).

Por meio do processo de avaliação é possível verificar a condição inicial do aluno, obter dados para prescrição e planejamento adequado e/ou individualizado das atividades de um programa de ensino, verificar se as metas pré-estabelecidas estão sendo alcançadas (Gorla e Campos, 2013), acompanhamento do progresso do aluno, além de poder estabelecer estágios evolutivos, seus níveis e propor recomendações para o desenvolvimento do avaliado (Mauerberg-deCastro, 2005)

Todavia, a literatura apresenta poucas opções de testes de habilidades em cadeira de rodas para avaliação motora de pessoas com deficiência física, sobretudo ao público infantil. Na maior parte dos casos, é possível identificar testes isolados para avaliação de habilidades específicas (Gorgatti e Bohme, 2003; Pasetto et al., 2011), fundamentos de modalidades esportivas adaptadas específicas (Brasile, 1990; Costa e Silva et al, 2010; Costa e Silva, 2011; Gorla et al., 2011), capacidades físicas e habilidades motoras (Winnick e Short, 2001), e habilidades motoras fundamentais (Zuvela et al., 2011; Herrmann e Seelig, 2016).

Um teste de habilidades em cadeira de rodas consiste em várias tarefas a serem executadas pelo candidato em condições padronizadas (Fliess-Douer et al., 2018). Tal ferramenta pode auxiliar na escolha adequada de habilidades a serem treinadas na reabilitação, bem como na avaliação de intervenções de treinamento. Além disso, um teste de habilidades em cadeira de rodas padronizado e aceito pode ser utilizado para desenvolver padrões de desempenho de habilidades em cadeira de rodas para indivíduos com diferentes níveis de deficiência (Fliess-Douer et al., 2018).

Devido à escassez de instrumentos voltados à avaliação das habilidades motoras e capacidades físicas de crianças com deficiência física em cadeira de rodas, surgiu a necessidade do estudo de Melo (2014). Esse estudo buscou verificar a influência de um programa de iniciação esportiva em cadeira de rodas voltado a crianças com deficiência física. No entanto, diante da dificuldade de identificar testes de avaliação, capazes de mensurar as variáveis do programa, percebeu-se a necessidade de construir um protocolo, denominado Protocolo Avaliativo de Iniciação Esportiva em Cadeira de Rodas (PAIE-CR).

Diante do exposto, a pesquisa em questão teve como objetivo: apresentar o Protocolo Avaliativo de Iniciação Esportiva em Cadeira de Rodas - PAIE-CR e verificar em quais áreas dos componentes motores da iniciação esportiva (habilidades motoras fundamentais e capacidades físicas) um programa de iniciação esportiva exerceu maior ou menor influência, antes e após a intervenção proposta.

\section{MÉTODO}

Trata-se de um estudo com enfoque qualiquantitativo, caracterizado como pesquisa de campo do tipo quantitativo-descritivo, voltada ao estudo de avaliação de programa (Marconi e Lakatos, 2008).

\section{PARTICIPANTES}

A amostra desse estudo foi constituída por três crianças com deficiência física, apresentando comprometimento nos membros inferiores. Os participantes estavam na faixa etária entre 6 a 10 anos de idade. 
Cabe informar que o estudo em questão foi aprovado pelo Comitê de Ética em Pesquisa da Universidade Federal de São Carlos - CEP/UFSCar, sob parecer de no72038, de acordo com as prerrogativas da resolução 466/12 da Comissão Nacional de Ética em Pesquisa (CONEP).

Os critérios de inclusão utilizados foram: crianças com deficiência física e comprometimento de membros inferiores; disponibilidade de horários para participação no programa. Como critérios de exclusão foram utilizados: participação anterior em outros programas de iniciação esportiva; presença de outros comprometimentos associados à deficiência física. No Quadro 1 pode ser encontrada a caracterização dos participantes da pesquisa. Para manter o anonimato dos participantes estes serão denominados da letra $P$ (participante) seguida de um numeral (ex.: P1).

\section{INSTRUMENTO DE COLETA DE DADOS}

O Protocolo Avaliativo de Iniciação Esportiva em Cadeira de Rodas - PAIE-CR (Melo, 2014), o qual passou pela validação do seu conteúdo (Melo, 2018). ${ }^{1}$

Esse instrumento foi elaborado com a finalidade de avaliar os componentes motores que influenciam no processo de iniciação esportiva em cadeira de rodas ${ }^{2}$ de pessoas com deficiência física, participantes de programas esportivos e/ou de educação física.

$O$ PAIE-CR é destinado a crianças, jovens e demais faixas etárias que se encaixem nas possibilidades do instrumento. Especificamente, pessoas com deficiência motora que possuam comprometimento nos membros inferiores, superiores e/ou tronco, tornando necessário e/ou possível o uso de cadeira de rodas durante o deslocamento na prática esportiva. Torna-se facultativa a utilização desse instrumento em outras populações, afinal, cabe aos avaliadores a criatividade e compreensão para melhor avaliar seus alunos.

Para elaboração do PAIE-CR foram reunidos testes de habilidades motoras e capacidades físicas, apresentados por meio dos fundamentos das modalidades esportivas em cadeira de rodas, tais como: propulsão e progressão na cadeira de rodas, arremesso, passe, recepção, drible e comportamentos como o controle de tronco, presentes no processo de iniciação, nas diversas atividades e situações diárias. 0 protocolo é composto por quatro grupos de testes diretamente relacionados com os componentes motores e seus

\footnotetext{
${ }^{1}$ O PAIE-CR foi fruto de uma pesquisa de doutoramento e por se tratar de um instrumento amplo, algumas informações não poderão ser apresentadas nesse momento. Porém, encontra-se disponível na web, na íntegra.

${ }^{2}$ As habilidades motoras fundamentais e capacidades físicas serão compreendidas nesse estudo como componentes motores da iniciação esportiva em cadeira de rodas.
}

respectivos fundamentos, totalizando nove testes, os quais foram adaptados e/ou especificamente criados.

No Quadro 2 encontra-se a caracterização dos componentes motores, fundamentos esportivos e testes presentes no protocolo (Melo, 2018):

Em sua construção, foram realizadas adaptações de determinados testes com vistas a obtenção de um instrumento para avaliação do programa de iniciação esportiva, por meio da avaliação e acompanhamento dos possíveis enriquecimentos motores dos participantes.

A seguir, encontra-se uma breve descrição dos grupos de testes componentes desse protocolo. Vide Quadro 3:

\section{PROCEDIMENTOS DE COLETA DE DADOS}

A pesquisa foi dividida em quatro etapas: 1) Anamnese 2) Avaliação inicial; 3) Período de realização da intervenção, correspondente ao programa de iniciação esportiva; 4) Avaliação final.

1a Etapa: Inicialmente os pais ou responsáveis dos participantes forneceram informações sobre os mesmos para melhor planejamento e controle da intervenção. Caso estes tivessem o interesse de participar da pesquisa, foi solicitado que assinassem o Termo de Consentimento Livre e Esclarecido - TCLE.

2a Etapa: A segunda etapa foi o momento de avaliação inicial por meio da aplicação do PAIE$C R$, realizada pré-intervenção, individualmente, para registro das condições iniciais dos participantes. Torna-se relevante ressaltar que os testes ocorreram no mesmo dia, com período de descanso entre os mesmos. Antes do início de cada teste encontra-se prevista a fase de vivência do teste. Além disso, não são ensinados nem estabelecidos padrões de movimento e/ ou fundamentos de modalidades esportivas específicas para a realização dos testes. Cabe destacar que os participantes utilizaram a mesma cadeira durante todo o processo.

3a Etapa: Caracterizada pelo período de intervenção, correspondente ao desenvolvimento do Programa de Iniciação Esportiva para crianças com deficiência física (Melo e Munster, 2016). O Programa de Iniciação Esportiva em Cadeira de Rodas para crianças com deficiência física teve como finalidade desenvolver os componentes motores na cadeira de rodas, por meio dos aspectos pedagógicos e educacionais utilizandose de brincadeiras e jogos pré-desportivos que possibilitem o ganho de independência para desenvolvimento nas atividades físicas e esportivas, bem como nas atividades da vida diária. 
Quadro 1. Caracterização dos Participantes.

\begin{tabular}{|lcccccc|}
\hline Nome & Gênero & Idade & Etiologia & Nível Funcional & Uso de Dispositivos & Freq. \\
\hline P1 & Masculino & 10 anos & Agenesia do corpo caloso & Diparesia & Andador & $100 \%$ \\
P2 & Masculino & 8 anos & Mielomeningocele / Hidrocefalia & Paraplegia(Torácica) & $\begin{array}{c}\text { Cadeira de rodas } \\
83,3 \%\end{array}$ \\
P3 & Feminino & 6 anos & Mielomeningocele / Hidrocefalia & Paraplegia (Lombar) & $\begin{array}{c}\text { Cadeira de rodas e/ou } \\
\text { Tutor e muletas }\end{array}$ & $91,6 \%$ \\
\hline
\end{tabular}

Freq: Frequência de participação no programa de iniciação esportiva. Fonte: Elaboração pelo autor.

Quadro 2. Caracterização do Protocolo Avaliativo de Iniciação Esportiva em Cadeira de Rodas.

\begin{tabular}{|c|c|c|}
\hline Componentes motores & Fundamentos & Testes \\
\hline \multirow{4}{*}{ Estabilizador } & \multirow{4}{*}{ Controle de tronco } & Controle de Troco (MELO, 2014) \\
\hline & & - Flexão anterior de Tronco; \\
\hline & & - Flexão lateral direita de Tronco; \\
\hline & & - Flexão lateral esquerda de Tronco \\
\hline & & - Velocidade (Adaptado de Winnick; Short, 2001) \\
\hline Locomotor & Deslocamento & $\begin{array}{l}\text { Agilidade (qualidade de deslocamento com mudanças de direção e sem perda } \\
\text { de velocidade) (Adaptado de Belasco; Silva, 1998; Pasetto et al, 2011). }\end{array}$ \\
\hline \multirow{4}{*}{ Manipulativo } & Arremesso & - Precisão de arremesso no alvo (Adaptado de Fonseca, 1995). \\
\hline & Passe; & - Precisão de arremesso no alvo (Precisão de arremesso na baliza (Melo, 2014) \\
\hline & Recepção & - Precisão de passe e recepção (Adaptado de Costa e Silva, 2011) \\
\hline & Drible & - Drible estático (Adaptado de Brasile, 1990) \\
\hline \multirow{2}{*}{ Combinados } & Drible & Teste de componentes motores combinados: \\
\hline & Deslocamento & - Drible em deslocamento (Adaptado de Brasile, 1990). \\
\hline
\end{tabular}

Fonte: Melo (2018)

O período de intervenção teve duração de oito semanas, três sessões/aulas por semana, perfazendo um total de 24 sessões em um período de dois meses. Cada uma das sessões teve duração média de 50 minutos. As aulas apresentavam a seguinte estrutura: Conversa inicial e alongamento; desenvolvimento; intervalo (hidratação e descanso) e volta a calma.

A duração do programa foi estabelecida a partir das características, potencialidades dos participantes e demais informações apresentadas na primeira etapa. Diante disso, compreendeu-se como um período mínimo de estímulos para dar início à quarta etapa.

4a Etapa: A última etapa refere-se ao momento de reaplicação do instrumento e registro das condições finais dos participantes pós-programa de iniciação esportiva. Os procedimentos foram os mesmos utilizados no momento da aplicação inicial. A duração média para aplicação completa do instrumento foi de $1 \mathrm{~h} 30$ minutos.

Para a análise dos dados, foi utilizada estatística descritiva.

\section{RESULTADOS E DISCUSSÃO}

Os resultados foram estruturados e apresentados por grupo de componente motor e seus respectivos testes, de maneira que os dados dos participantes serão anunciados de forma individualizada, mostrando os resultados das aplicações antes e após o programa de iniciação esportiva.

Inicialmente, serão apresentados os testes de componentes de estabilização, os quais são compostos por três testes, são eles: Flexão anterior de tronco, flexão lateral direita de tronco e flexão lateral esquerda de tronco. Os resultados das aplicações encontram-se na Tabela 1.

Ao analisar os resultados dos testes de estabilização, por meio do controle de tronco, pôde-se notar que todos os participantes obtiveram melhora no aumento da amplitude dos testes, uma vez que P1, P2 e P3 apresentaram evoluções nos índices e, quando essas evoluções não foram perceptíveis, os índices alcançados após o período de intervenção permaneceram os mesmos.

O controle de tronco é fundamental para qualquer indivíduo, sobretudo para aqueles que fazem uso da cadeira de rodas. Dentro do processo de iniciação esportiva, buscou-se possibilitar aos participantes do estudo o desenvolvimento das capacidades de estabilização, compreendendo-as 
Quadro 3. Descrição dos testes e finalidades por grupo de componentes.

\begin{tabular}{|c|c|}
\hline & Caracterização dos testes presentes no PAIE-CR \\
\hline Obs.: Antes do iníci & o teste o participante passará pela fase de vivência do teste, composto por três tentativas. \\
\hline Testes & Finalidade e características \\
\hline $\begin{array}{l}\text { Controle de tronco } \\
\text { (Flexão anterior de } \\
\text { Tronco }\end{array}$ & $\begin{array}{l}\text { Avaliar a capacidade de controle de tronco com base no grau de amplitude da flexão anterior } \\
\text { realizada de forma independente pelo participante. A unidade de medida é em graus. O participante } \\
\text { não poderá fazer a recuperação do movimento com auxílio dos braços e mãos. O participante poderá } \\
\text { utilizar os recursos de compensação que utiliza diariamente (ex.: órteses e próteses). O participante } \\
\text { terá três tentativas, será considerado o maior índice alcançado }\end{array}$ \\
\hline & 7 \\
\hline $\begin{array}{l}\text { Controle de tronco } \\
\text { (Flexão Lateral de } \\
\text { Tronco) }\end{array}$ & $\begin{array}{l}\text { Avaliar a capacidade de controle de tronco com base no grau de amplitude da flexão lateral (direita } \\
\text { e esquerda), realizada de forma independente pelo participante. A unidade de medida é em graus. } \\
\text { O participante não poderá fazer a recuperação do movimento com auxílio dos braços e mãos. O } \\
\text { participante poderá utilizar os recursos de compensação que utiliza diariamente (ex.: órteses e } \\
\text { próteses). O participante terá três tentativas para cada lado, serão considerados os maiores índices } \\
\text { alcançados em cada lado. }\end{array}$ \\
\hline & Fonte: Melo (2018) \\
\hline $\begin{array}{l}\text { Deslocamento } \\
\text { (Velocidade) }\end{array}$ & $\begin{array}{l}\text { Avaliar a capacidade de deslocamento em cadeira de rodas do participante em velocidade, obtendo } \\
\text { o tempo gasto para percorrer o trajeto de } 40 \text { metros. O participante não poderá ultrapassar a linha de } \\
\text { início já em aceleração. O participante terá cinco tentativas, duas para reconhecimento do percurso } \\
\text { e as três seguintes serão consideradas válidas para o teste. O participante terá três minutos para } \\
\text { descanso entra as tentativas, a fim de se evitar efeitos de fadiga. }\end{array}$ \\
\hline & $\underset{\text { Fonte: Melo (2018) }}{\rightarrow}$ \\
\hline $\begin{array}{l}\text { Deslocamento } \\
\text { (Agilidade) }\end{array}$ & $\begin{array}{l}\text { Avaliar a capacidade de deslocamento em cadeira de rodas em agilidade do participante, } \\
\text { compreendendo que a agilidade possibilita mudança de direção sem perda de velocidade e ritmo, } \\
\text { obtendo o tempo gasto para percorrer o trajeto. O participante não poderá ultrapassar a linha de } \\
\text { início já em aceleração. O participante terá cinco tentativas, duas para reconhecimento do percurso } \\
\text { e as três seguintes serão consideras válidas para o teste. O participante terá três minutos para } \\
\text { descanso entre as tentativas, a fim de se evitar efeitos de fadiga. }\end{array}$ \\
\hline
\end{tabular}


Continuação...

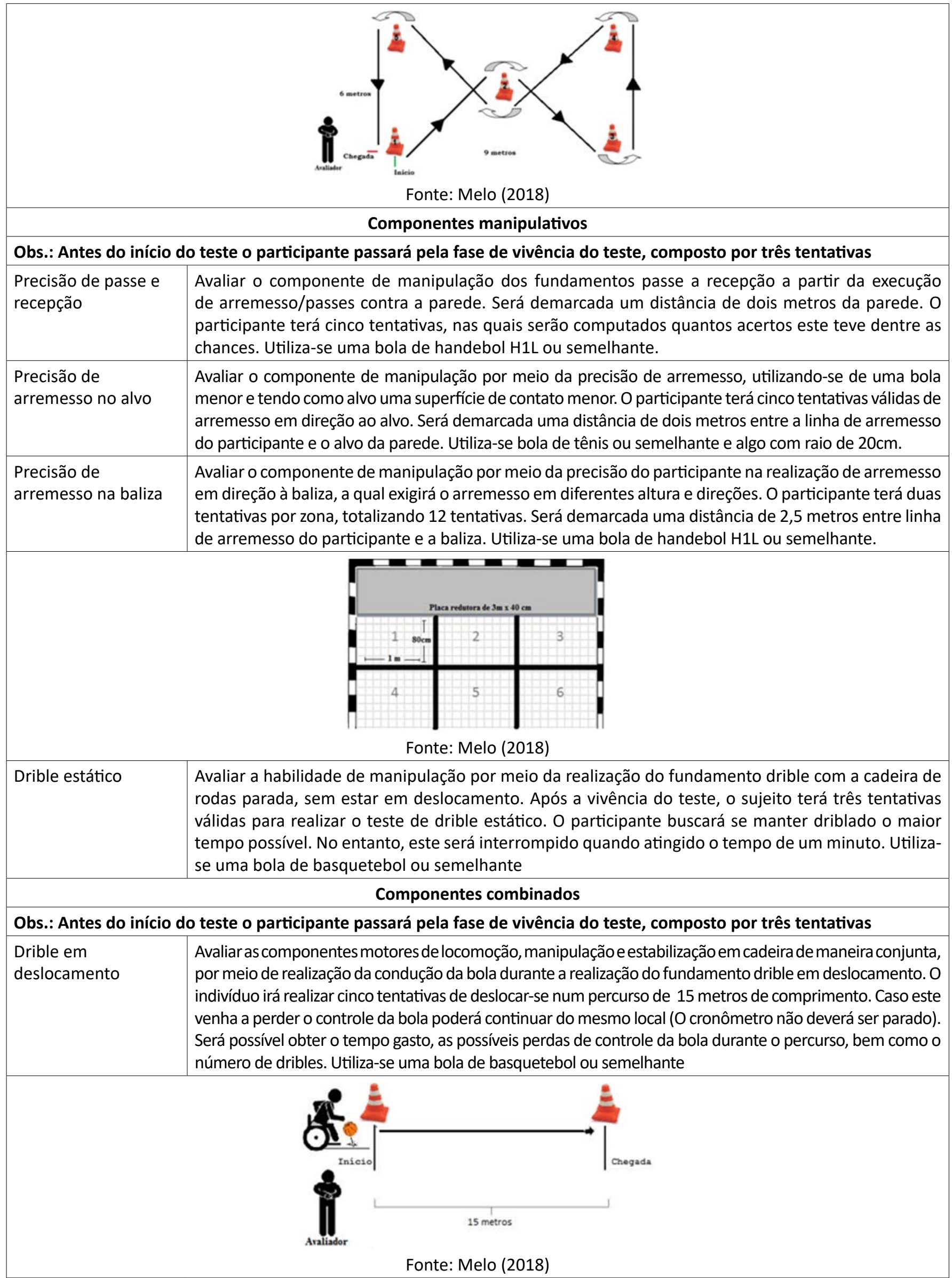


Tabela 1. Descrição dos resultados nos testes de componentes motores estabilizadores

\begin{tabular}{|c|c|c|c|c|c|c|c|c|c|}
\hline \multirow{4}{*}{$\begin{array}{l}\text { Unid. } \\
\text { Part }\end{array}$} & \multicolumn{9}{|c|}{ Teste de componentes de estabilização } \\
\hline & \multicolumn{3}{|c|}{ Flexão anterior de tronco } & \multicolumn{3}{|c|}{ Flexão lateral direita } & \multicolumn{3}{|c|}{ Flexão lateral esquerda } \\
\hline & \multicolumn{2}{|c|}{ Graus } & \multirow{2}{*}{$\begin{array}{c}\% \\
\text { Melhora }\end{array}$} & \multicolumn{2}{|c|}{ Graus } & \multirow{2}{*}{$\begin{array}{c}\% \\
\text { Melhora }\end{array}$} & \multicolumn{2}{|c|}{ Graus } & \multirow{2}{*}{$\begin{array}{c}\% \\
\text { Melhora }\end{array}$} \\
\hline & Pré & Pós & & Pré & Pós & & Pré & Pós & \\
\hline P1 & $55^{\circ}$ & $75^{\circ}$ & 22.2 & $10^{\circ}$ & $20^{\circ}$ & 11.1 & $20^{\circ}$ & $30^{\circ}$ & 11.1 \\
\hline P2 & $40^{\circ}$ & 40 은 & 0 & $15^{\circ}$ & $20^{\circ}$ & 5.5 & $25^{\circ}$ & $30^{\circ}$ & 5.5 \\
\hline P3 & $90^{\circ}$ & $80^{\circ}$ & -11.1 & $20^{\circ}$ & $30^{\circ}$ & 11.1 & $20^{\circ}$ & $30^{\circ}$ & 11.1 \\
\hline
\end{tabular}

Part. $=$ Participantes; Unid. $=$ Unidade de medida; $X=$ Sujeito não realizou o teste; \% Melhora = Percentual de melhora. Fonte: Elaborado pelo autor.

como habilidades essenciais a serem trabalhadas num processo de iniciação esportiva. Ressalta-se que esse comportamento é fundamental para usuários de cadeira de rodas, principalmente na execução das atividades de vida diária - que incluem a higiene pessoal, vestir-se e alimentar-se, e de posições de alívio de pressão visando a prevenção de escaras (Peres et al., 2007).

Foi possível encontrar semelhanças com o estudo realizado por Peres et al. (2007), onde os autores visaram avaliar a contribuição de um treinamento baseado em dança para o ganho de amplitude de movimento de tronco em paraplégicos. Ainda que o estudo tenha outra conformação e se utilize de diferentes métodos, os resultados alcançados nas amplitudes dos testes são semelhantes e obtiveram incrementos nos componentes motores após as intervenções.

Referente aos componentes de locomoção, compreendidos pelos testes de velocidade e agilidade. Para melhor compreensão dos índices de evolução dos participantes, os dados encontram-se descritos no Tabela 2.

Tabela 2. Descrição dos resultados nos testes de componentes motores de locomoção.

\begin{tabular}{lcccc}
\hline & \multicolumn{3}{c}{ Teste de componentes locomotores } \\
\cline { 2 - 5 } & \multicolumn{2}{c}{ Teste de velocidade } & \multicolumn{2}{c}{ Teste de Agilidade } \\
\hline Unid. & \multicolumn{2}{c}{ Tempo (Segundos) } & \multicolumn{2}{c}{ Tempo (Segundos) } \\
\hline Part & Pré & Pós & Pré & Pós \\
P1 & $160 s$ & $34 s$ & $148 s$ & $42 \mathrm{~s}$ \\
P2 & $26 \mathrm{~s}$ & $19 \mathrm{~s}$ & $40 \mathrm{~s}$ & $26 \mathrm{~s}$ \\
P3 & $47 \mathrm{~s}$ & $28 \mathrm{~s}$ & $\mathrm{X}$ & $\mathrm{X}$ \\
\hline
\end{tabular}

Part. = Participantes; Unid. $=$ Unidade de medida; $X=$ Sujeito não realizou o teste. Fonte: Elaborado pelo autor.

No teste de "velocidade", além de uma redução do tempo para execução do mesmo, percebeu-se também avanços qualitativos dos participantes, uma vez que os mesmos obtiveram evoluções no seu controle sobre a cadeira, proporcionando menor tempo na execução das tarefas. O mesmo ocorreu no teste de "agilidade" para P1 e P2. No caso de P3, também apresentou melhoras no seu desempenho na cadeira (execução dos giros, mudança brusca de direção, curvas), ainda que a participante não conseguira compreender o percurso do teste.

P1 era o único que não fazia uso da cadeira de rodas diariamente, o mesmo utilizava-se de um andador, dispositivo que limitava demasiadamente seus movimentos de tronco e de marcha. Acreditase que a utilização da cadeira de rodas possibilitou a experimentação de movimentos e o fortalecimento do tronco. 0 mesmo passou a mudar seus hábitos, a realizar a marcha com maior facilidade, adquirir maior equilíbrio e movimentos de tronco antes não realizados, diminuiu a utilização do andador, estimulando-o a se locomover com maior independência.

Uma grande proporção de crianças usam cadeira de rodas diariamente para se locomover. Em adultos, vários estudos relataram a importância das habilidades de mobilidade em cadeira de rodas para superar problemas de mobilidade e melhorar a participação (MacPhee et al., 2004; Routhier et al., 2012; Sol et al, 2017). Em crianças, são poucas as evidências, a exemplo dos estudo de intervenção de Sawatzki et al (2012); fatores associados aos níveis de atividade física (Bloemen et al., 2015); e o estudo de desenvolvimento de um teste de habilidades de mobilidade em cadeira de rodas para crianças e jovens (Sol et al., 2017).

Gorgatti e Böhme (2003) ressaltam que a capacidade de aceleração da cadeira de rodas a partir da posição parada é determinada por três componentes principais: a configuração da cadeira de rodas (Batavia et al., 2001; Di Marco et al., 2003), a potência, a técnica de propulsão do usuário atrelada ao ajuste da cadeira de rodas e as características do praticante. Portanto, acredita-se que o programa proporcionou aos participantes aprimoramento das suas capacidades de potência, ajuste à cadeira, considerando o maior controle de tronco na execução das fases de propulsão e recuperação, culminando na diminuição do tempo da tarefa. 
Referente aos componentes manipulativos, esses são compostos por quatro testes, são eles: teste de arremesso à baliza, teste de arremesso ao alvo, teste de passe e recepção e teste de drible estático. Os resultados das aplicações pré e pós intervenção encontram-se na Tabela 3.

Em relação aos componentes de manipulação, além dos ganhos quantitativos, os participantes demonstraram ganhos em termos de qualidade do movimento (postura para execução dos arremessos, adaptação à cadeira de rodas e menor tempo para execução dos testes).

No teste de "arremesso na baliza" os participantes apresentaram evoluções e/ou índices elevados. Nos casos de P1 e P3, esses atingiram médias maiores após a aplicação do programa, acompanhadas de claras melhoras qualitativas de aprimoramento desse fundamento. No caso específico de P2, o participante obteve escores muito elevados no pré-teste e também no pós-teste, porém obteve um número de acertos inferior ao primeiro. É importante ressaltar que P2 foi o participante que apresentou melhor desempenho, desde o início, e que o mesmo apresentou grande evolução na execução qualitativa desse fundamento. No teste de "arremesso ao alvo", todos os participantes apresentaram evoluções quantitativas e qualitativas, semelhantemente ao teste anterior.

Dentre os componentes que devem ser controlados e são necessários para execução do arremesso e/ ou lançamento, pode-se destacar elementos como profundidade e ângulos de lançamentos como fatores importantes para a execução do arremesso ou lançamento, isso aliado à orientação corporal, gestual e dinâmica de movimento facilita ganho de espaço para execução do fundamento (Gorla et al., 2010). Considerando ainda a utilização de tais componentes na cadeira de rodas, deve-se compreender como um processo dinâmico, e por vezes, individual.

Diante disso, a escolha das atividades a serem propostas para o aprimoramento do arremesso deve ser cautelosa. Tendo em vista que
O desenvolvimento do arremesso não é linear ou prescritivo; em vez disso, ele é dinâmico e variável por natureza. É importante que os professores compreendam esse ponto crítico. Quando selecionam tarefas para seus alunos, podem provocar o surgimento de um padrão mais avançado de arremesso, desde que considerem as restrições individuais e modifiquem as restrições da tarefa e do ambiente para gerar essa performance (Gallahue et al., 2013, p.218).

Portanto, para elaboração e planejamento das atividades foram consideradas as variadas possibilidades econdições dos participantes, utilizando-se de diferentes padrões de movimento, sem maiores direcionamentos para as especificidades das modalidades esportivas.

No teste de "precisão de passe e recepção", todos os participantes apresentaram índices maiores após a intervenção, e passaram a realiza o fundamento com maior facilidade e segurança, em alguns casos executaram o teste com apenas uma das mãos. Nesse sentido,

As crianças precisam de várias habilidades a fim de conseguir pegar uma bola, incluindo a coordenação olho-mão, a habilidade de rastrear o objeto com os olhos, antecipar a sua trajetória de modo consistente e intercepta-lo, a consciência perceptiva e a manipulação fina dos dedos de acordo com a dinâmica de voo do objeto. (Gallahue et al., 2013, p.226).

Como estratégia para desenvolvimento de tais fundamentos, valeu-se do princípio que "as atividades simples devem levar às mais complexas, e as atividades conhecidas devem levar ao desconhecido" (Bompa, 2002). Esse modo de conduzir as atividades proporcionou aos participantes que compreendessem os objetivos a serem alcançados e que obtivessem evoluções de maneira progressiva.

O teste de "drible estático" apresentou variados resultados. Os participantes se manifestaram de diferentes maneiras, ficando clara a influência de aspectos como: adaptação à cadeira de rodas, etiologia, vivências anteriores. P1 e P2 obtiveram evoluções

Tabela 3. Descrição dos resultados nos testes de componentes motores de manipulação

\begin{tabular}{|c|c|c|c|c|c|c|c|c|}
\hline \multirow{4}{*}{$\begin{array}{l}\text { Unid. } \\
\text { Part }\end{array}$} & \multicolumn{8}{|c|}{ Teste de componentes manipulativos } \\
\hline & \multicolumn{2}{|c|}{ Arremesso à baliza } & \multirow{2}{*}{\multicolumn{2}{|c|}{$\begin{array}{l}\text { Arremesso ao alvo } \\
\text { № de conversões }\end{array}$}} & \multirow{2}{*}{\multicolumn{2}{|c|}{$\begin{array}{l}\text { Passe e recepção } \\
\text { № de conversões }\end{array}$}} & \multicolumn{2}{|c|}{ Drible estático } \\
\hline & \multicolumn{2}{|c|}{ № de conversões } & & & & & \multicolumn{2}{|c|}{ № de execuções/min } \\
\hline & Pré & Pós & Pré & Pós & Pré & Pós & Pré & Pós \\
\hline P1 & 2 & 5 & 1 & 2 & 0 & 2 & 2 & 11 \\
\hline $\mathrm{P} 2$ & 11 & 9 & 0 & 1 & 4 & 5 & 6 & 69 \\
\hline P3 & 4 & 6 & 0 & 1 & 0 & 3 & $x$ & $x$ \\
\hline
\end{tabular}

Part. = Participantes; Unid. = Unidade de medida; $X$ = Sujeito não realizou o teste. Fonte: Elaborado pelo autor. 
nesse fundamento, em graus variados. No caso de P1, inicialmente apresentava maiores dificuldades. Ainda assim, o participante atingiu índices significativos, apresentando maior segurança para realização do teste e melhora da sua percepção espaço-temporal para controle da bola. P2 apresentou melhora grandiosa em todos os aspectos. No caso específico de P3, apresentou maiores dificuldades em sua adaptação à cadeira de rodas nesse fundamento e não realizou o teste.

São variados os componentes manipulativos, podese afirmar que é durante essa fase do desenvolvimento que as crianças apresentam maior receptividade para o aprendizado e aprimoramento de tais habilidades (Gallahue et al., 2013; Higgs, 2011). Referindo-se às habilidades coordenativas Galatti e Paes (2007) ressaltam que no início da puberdade a criança possui maiores facilidades para aprimorar essas habilidades,

em razão da maior excitabilidade cerebral, melhoria das funções visuais e acústicas, crianças nesse período possuem maior facilidade de assimilar informações, sendo este momento especialmente propício para ensinar novas coisas, variar os estímulos e propiciar desafios, possibilitando que se criem novos movimentos e promova à criança um amplo repertório motor nesse período. (Galatti e Paes, 2007, p.78).

Tais afirmativas corroboram com Gallahue et al. (2013) quando afirmam que esse é um período agitado, momento de muitas descobertas, onde as crianças encontram-se submersas em suas habilidades motoras, as quais são ampliadas em passo acelerado. Nesse sentido, é importante que todas as crianças, inclusive as com deficiência, desenvolvam as habilidades motoras fundamentais antes que as habilidades esportivas específicas sejam introduzidas (Higgs, 2011). Diante do exposto, compreende-se a necessidade de desenvolvimento dessas habilidades como uma prioridade a ser trabalhada com todas as crianças, e não somente um privilégio das crianças sem deficiência.

Referente ao teste de componentes combinados, este é composto por uma tarefa complexa, para realizá-lo é necessário coordenar o movimento da bola juntamente às propulsões na cadeira de rodas de forma alternada. Portanto exige a utilização dos componentes de locomoção, manipulação e estabilização, simultaneamente. Nesse teste, inicialmente todos os participantes demonstraram dificuldades, não conseguindo realizá-lo. Após o período de intervenção, apenas $\mathrm{P} 2$ conseguiu realizar o teste, realizando 31 dribles durante o percurso em 21.31 segundos, sem perdas de controle. Os demais participantes, mesmo após o período de intervenção, não conseguiram realizá-lo.
A criança no estágio de iniciação, deve participar de programas bem estruturados para o desenvolvimento de competências, com ênfase no divertimento em busca de um desenvolvimento esportivo geral (Bompa, 2002; Higgs, 2011). Tais afirmativas reforçam a necessidade do desenvolvimento de um programa de iniciação esportiva para crianças com deficiência física não direcionado à uma única modalidade esportiva, e sim às similaridades existentes entre essas, apresentadas por meio dos fundamentos específicos agrupados nesse estudo como componentes motores, apoiando-se na riqueza dos jogos pré-desportivos e brincadeiras para atingir os objetivos propostos (Melo e Munster, 2016).

Assim, para além das evoluções dos fundamentos na realização das atividades lúdicas e pré-desportivas, tais atividades passaram a lhes possibilitar melhores resultados para a efetivação das atividades diárias com maior independência.

Quanto às limitações do estudo, vê-se a necessidade de ampliação da amostra para um número mais representativo. Ainda que o instrumento tenha passado pelo processo de validação de conteúdo, torna-se essencial a realização de novos estudos, tais como: a validação dos critérios de fidedignidade intraavaliadores e inter-avaliadores, bem como os demais aspectos atrelados à confiabilidade do instrumento.

\section{CONCLUSÃO}

Ainda são poucos os instrumentos de avaliação voltados ao público em cadeira de rodas, sobretudo quando referente ao público infantil. Diante disso, foram estabelecidos critérios e parâmetros de avaliação para elaboração de um instrumento, utilizado para a avaliação de um programa de iniciação esportiva em cadeira de rodas, utilizado, nesse momento, com crianças com deficiência física. O protocolo apresentado possibilitou por meio das avaliações a obtenção de parâmetros iniciais dos componentes motores atrelados à iniciação esportiva (habilidades motoras fundamentais e capacidades físicas) e demais comportamentos, após o programa. Foi possível verificar que o período de intervenção influenciou em todos os componentes motores.

Dentre suas potencialidades, apresenta-se a possibilidade de verificar a condição inicial efetiva para acompanhar os níveis de desenvolvimento dos componentes motores de crianças e/ou jovens e adultos com deficiência física que se encaixem nas possibilidades do instrumento, em variados contextos (inclusivo e/ou segregado); auxiliar na elaboração de programas de ensino dessa natureza e avaliar o progresso global e individual em cada grupo de componentes motores. Além disso, pode-se utilizar 
para fins de pesquisa, como protocolo de testes voltado aos aspectos inerentes à iniciação esportiva em cadeira de rodas e suas nuances.

Diante do exposto, compreende-se que essas são habilidades e capacidades a serem estimuladas em todas as pessoas, e fundamentalmente as crianças, com e sem deficiência, e não ser um privilégio de uma dessas. Por fim, pode-se concluir que quando são ofertadas oportunidades de vivências motoras às crianças com deficiência física por meio de atividades lúdicas, jogos e práticas esportivas, estas passam a se desenvolver.

\section{AGRADECIMENTOS}

Os autores gostariam de agradecer e destacar que a pesquisa em questão obteve o essencial apoio das agências de fomento: Conselho Nacional de Desenvolvimento Científico e Tecnológico (CNPq) pela concessão de bolsa de mestrado (2012-2014) e à Coordenação de Aperfeiçoamento de Pessoal de Nível Superior (Capes) pela concessão de bolsa de doutorado (2014-2015).

\section{REFERÊNCIAS}

Batavia M, Batavia Al, Friedman R. Changing chairs: anticipating problems in prescribing wheelchairs. Disability and rehabilitation 2001; 23 (12): 539-548.

Belasco Junior D, SILVA AC. Consistência dos resultados do teste de corrida em ziguezague de Barrow (modificado) em jogadores de basquetebol em cadeira de rodas. INTERNATIONAL CONGRESS OF MOTOR REHABILITATION; 1998 Oct 5-8; Águas de Lindóia, SP; 1998.

Bloemen MA, Verschuren O, van Mechelen C, Borst HE, de Leeuw AJ, van der Hoef $M$, de Groot JF. Personal and environmental factors to consider when aiming to improve participation in physical activity in children with Spina Bifida: a qualitative study. BMC Neurol. 2015;15:11.

Bompa TO. Treinamento total para jovens campeões. Barueri, SP: Manole; 2002.

Brasile F. Performance avaluation of wheelchair athletes: more than a disability classification level issue. Adapted Physical Activity Quarterly 1990;.(7): 289-297.

Costa e Silva AA, Gorla JI, Calegari DR, Costa LT. Adaptação de uma bateria de testes para handebol em cadeira de rodas. R. bras. Ci e Mov 2010; 18 (3): 73-80.

Costa e Silva AA. Validação de uma bateria de testes de habilidades motoras para atletas de handebol em cadeira de rodas. [Dissertação]. Campinas,SP: Faculdade de Educação Física, Universidade Estadual de Campinas - Unicamp; 2011.

Dalhousie University: Wheelchair Skills Program. 2012. [Acesso em: 30, Set, 2018]. Disponível em: http://www. wheelchairskillsprogram.ca/.

Di Marco A, Russel M, Masters M. Standards for wheelchair prescription. Australian Occupational Therapy Journal 2003; 50 (1): 30-9.

Fliess-Douer O, Vanlandewijck YC, Manor GL, Van Der Woude LHV. A systematic review of wheelchair skills tests for manual wheelchair users with a spinal cord. injury: towards a standardized outcome measure. Clin Rehabil 2010; 24: 867.
Fonseca V. Manual de observação psicomotora. Porto Alegre, RS: Artes Médicas; 1995.

Galatti LR, Paes RR. Pedagogia do esporte: iniciação ao basquetebol. Hortolândia, SP: UNASP; 2007.

Gallahue DL, Ozmun JC, Goodway JD. Compreendendo o desenvolvimento motor: bebês, crianças, adolescentes e adultos. 7a ed. Porto Alegre, RS: AMGH; 2013.

Giesbrecht EM, Miller WC, Mitchell IM, Woodgate RL. Development of a Wheelchair Skills Home Program for Older Adults Using a Participatory Action Design Approach. BioMed Research International; 2014: 1-13.

Gorgatti MG, Böhme MTS. Autenticidade científica de um teste de agilidade para indivíduos em cadeira de rodas. Rev. paul. Educ. Fís. 2003; 17 (1): 41-50.

Gorgatti MG, Costa RF. Atividade Física Adaptada: qualidade de vida para pessoas com necessidades especiais. $3^{a}$ ed. Barueri, SP: Manole; 2013.

Gorla JI, Calegari DR, Carminato RA, Costa e Silva AA. Iniciação ao treinamento do Handebol em Cadeira de Rodas. In: Calegari, DR, Gorla JI, ARAÚJO PF. Handebol em Cadeira de Rodas: regras e treinamento. São Paulo: Phorte; 2010. p. 63-84.

Gorla JI, Costa e Silva AA, Costa LT, Campos LFCC. Validação da bateria "Beck" de testes de habilidades para atletas brasileiros de "rugby" em cadeira de rodas. Rev. bras. Educ. Fís. Esporte 2011; 25(3): 473-486.

Gorla Jl, Campos LFCC. Processo de avaliação motora em Educação Física Adaptada. In: Gorla, J. I. Educação Física Adaptada: o passo a passo da avaliação. 2 ed. São Paulo, SP: Forte; 2013. p. 27-49.

Herrmann C, Seelig H. Structure and Profiles of Basic Motor Competencies in the Third Grade-Validation of the Test Instrument MOBAK-3. Perceptual and Motor Skills. 2016; 124(1):5-20.

Higgs C. Integration of Persons with a Disability in Canadian Sport. Canadian Sport for Life: Special report Canadian sport for life for athletes with a disability. 2011; Canadá, p.3-15.

MacPhee $\mathrm{AH}$ et al. Wheelchair skills training program: a randomized clinical trial of wheelchair users undergoing initial rehabilitation1. Arch Phys Med Rehabil. 2004; 85(1):41-50.

Marconi MA, Lakatos EM. Técnicas de Pesquisa. 7ạ ed. São Paulo: Atlas; 2008.

Mauerberg-deCastro E. Atividade Física: adaptada. Ribeirão Preto, SP: Tecmedd; 2005.

Melo FAP. Influência de um programa de iniciação esportiva em crianças com deficiência física. [Dissertação]. São Carlos, SP: Programa de Pós-Graduação em Educação Especial, Universidade Federal de São Carlos-UFSCar; 2014.

Melo FAP. Construção, validação de conteúdo e aplicação do protocolo avaliativo de iniciação esportiva em cadeira de rodas. [Tese]. São Carlos, SP: Programa de Pós-Graduação em Educação Especial, Universidade Federal de São CarlosUFSCar; 2018.

Melo FAP, Munster MAV. Iniciação esportiva em cadeira de rodas: estruturação de um programa para crianças com deficiência física. Pensar a Prática 2016; 19 (1): 68-80.

Ozturk A, Ucsular FD. "Effectiveness of a Wheelchair " Skills Training Programme for community-living users of manual wheelchairs in Turkey: a randomized controlled trial," Clinical Rehabilitation 2011; 25 (5): 416-424.

Pasetto CVR et al. Validação do teste de agilidade em ziguezague para crianças com deficiência física. R. da Educação Física/UEM. 2011; 22 (2): 173-180. 
Peres MS, Mello F, Gonçalves CA. Efeitos da dança em cadeira de rodas no controle de movimentos de tronco em paraplégicos. Arquivos em movimento. 2007; 3 (3): 56-66.

Routhier F et al. Efficacy and Retention of the French-Canadian Version of the Wheelchair Skills Training Program for Manual Wheelchair Users: A Randomized Controlled Trial. Arch Phys Med Rehabil. 2012;93(6):940-8.

Sawatzky B, Rushton PW, Denison I, McDonald R. Wheelchair skills training programme for children: a pilot study. Aust Occup Ther J. 2012; 59(1):2-9.
Sol ME et al. Development of a wheelchair mobility skills test for children and adolescents: combining evidence with clinical expertise, BMC Pediatrics. 2017; 17(1):51.

Winnick JP, Short FX. Testes de aptidão física para jovens com necessidades especiais. Tamboré, SP: Editora Manole; 2001.

Zuvela F, Bozanic A, Miletic D. POLYGON - A New Fundamental Movement Skills Test for 8 Year Old Children: Construction and Validation, J Sports Sci Med. 2011; 10(1): 157-163. 